\title{
From the desktop to the grid: scalable bioinformatics via workflow conversion
}

\author{
Luis de la Garza ${ }^{1}$, Johannes Veit ${ }^{1}$, Andras Szolek ${ }^{1}$, Marc Röttig ${ }^{1}$, Stephan Aiche ${ }^{2}$, Sandra Gesing ${ }^{3}$, \\ Knut Reinert ${ }^{2}$ and Oliver Kohlbacher ${ }^{1}$
}

\begin{abstract}
Background: Reproducibility is one of the tenets of the scientific method. Scientific experiments often comprise complex data flows, selection of adequate parameters, and analysis and visualization of intermediate and end results. Breaking down the complexity of such experiments into the joint collaboration of small, repeatable, well defined tasks, each with well defined inputs, parameters, and outputs, offers the immediate benefit of identifying bottlenecks, pinpoint sections which could benefit from parallelization, among others. Workflows rest upon the notion of splitting complex work into the joint effort of several manageable tasks.

There are several engines that give users the ability to design and execute workflows. Each engine was created to address certain problems of a specific community, therefore each one has its advantages and shortcomings.

Furthermore, not all features of all workflow engines are royalty-free - an aspect that could potentially drive away members of the scientific community.

Results: We have developed a set of tools that enables the scientific community to benefit from workflow interoperability. We developed a platform-free structured representation of parameters, inputs, outputs of command-line tools in so-called Common Tool Descriptor documents. We have also overcome the shortcomings and combined the features of two royalty-free workflow engines with a substantial user community: the Konstanz Information Miner, an engine which we see as a formidable workflow editor, and the Grid and User Support Environment, a web-based framework able to interact with several high-performance computing resources. We have thus created a free and highly accessible way to design workflows on a desktop computer and execute them on high-performance computing resources.

Conclusions: Our work will not only reduce time spent on designing scientific workflows, but also make executing workflows on remote high-performance computing resources more accessible to technically inexperienced users. We strongly believe that our efforts not only decrease the turnaround time to obtain scientific results but also have a positive impact on reproducibility, thus elevating the quality of obtained scientific results.
\end{abstract}

Keywords: Workflow, Interoperability, KNIME, Grid, Cloud, Galaxy, gUSE

\section{Background}

The importance of reproducibility for the scientific community has been a topic lately discussed in both highimpact scientific publications and popular news outlets $[1,2]$. To be able to independently replicate results-be it for verification purposes or to further advance researchis important for the scientific community. Therefore, it

*Correspondence: delagarza@informatik.uni-tuebingen.de

${ }^{1}$ Center for Bioinformatics and Dept. of Computer Science, University of

Tübingen, Sand 14, 72070 Tübingen, Germany

Full list of author information is available at the end of the article is crucial to structure an experiment in such a way that reproducibility could be easily achieved.

Workflows are structured, abstract recipes that help users construct a series of steps in an organized way. Each step is a parametrised specific action that receives some input and produces some output. The collective execution of these steps is seen as a domain-specific task.

With the availability of biological big data, the need to represent workflows in computing languages has also increased [3]. Scientific tasks such as genome comparison, mass spectrometry analysis, protein-protein interaction, just to name a few, access extensive datasets. Currently, 
a vast number of workflow engines exist [4-8] and each of these technologies has amassed a considerable user base. These engines support, in some way or another, the execution of workflows on distributed high-performance computing (HPC) resources (e.g., grids, clusters, clouds, etc.), thus allowing speedier obtention of results. A wise selection of a workflow engine will shorten the time spent between workflow design and retrieval of results.

\section{Workflow engines}

Galaxy [6] is a free web-based workflow system with several pre-installed tools for data-intensive biomedical research. Inclusion of arbitrary tools is reduced to the trivial task of creating ToolConfig [9] files, which are Extensible Markup Language documents (XML). The Galaxy project also features a so-called toolshed [10], from which tools can be obtained and installed on Galaxy instances. At the time of writing Galaxy's toolshed featured 3470 tools. However, we have found that Galaxy lacks extended support for popular workload managers and middlewares.

Taverna [7] offers an open-source and domainindependent suite of tools used to design and execute scientific workflows, helping users to automate and pipeline processing of data coming from different web services. At the time of writing Taverna features more than 3500 services available on startup and it also provides access to local and remote tools. Taverna allows users to track results and data flows with great granularity, since it implements the Open Provenance Model standard (OPM) [11]. A very attractive feature of Taverna is the ability to share workflows via the myExperiment research environment [12].

The Konstanz Information Miner Analytics Platform (KNIME Analytics Platform) [4, 13] is a royalty-free engine that allows users to build and execute workflows using a powerful and user-friendly interface. The KNIME Analytics Platform comes preloaded with several ready-to-use tasks (called KNIME nodes) that serve as the building stones of a workflow. It is also possible to extend the KNIME Analytics Platform by either downloading community nodes or building custom nodes using a well-documented process $[14,15]$. Workflows executed on the KNIME Analytics Platform are limited to run on the same personal computer on which it has been installed, thus rendering it unsuitable for tasks with highmemory or high-performance requirements. KNIME is offered in two variants able to execute workflows on distributed HPC resources: KNIME Cluster Execution [16], and KNIME Server [17]. These two suites are, however, royalty-based-an aspect that might shy away users of the scientific community.

The grid and cloud User Support Environment (gUSE) offers an open-source, free, web-based workflow platform able to tap into distributed HPC infrastructures [5]. gUSE entails a set of components and services that offers access to distributed computing interfaces (DCI). The Web Services Parallel Grid Runtime and Developer Environment Portal (WS-PGRADE) component acts as the graphical user interface. This web-based portal is a series of dynamically generated web pages, through which users can create, execute, and monitor workflows. WS-PGRADE communicates with internal gUSE services (e.g., Workflow Interpreter, Workflow Storage, Information Service) using the Web Services Description Language (WSDL) [18]. Passing documents in the WSDL format between its components allows gUSE services to interact with other workflow systems. Figure 1 shows the three-tiered architecture of gUSE. This complex and granular architecture of gUSE enables administrators to distribute the installation of gUSE across resources. A typical set-up is to install WS-PGRADE on a dedicated web server, while installing other services and components on more powerful computers.

In order to provide a common workflow submission Application Programming Interface (API), gUSE channels workflow-related requests (i.e., start, monitor, cancel a job on a DCI) through the DCI Bridge component [19]. The DCI Bridge is fully compatible with the Job Submission Description Language (JSDL) [20], thus enabling other workflow management systems to interact with it in order to benefit from gUSE's flexibility and DCI support. The DCI Bridge contains so-called DCI Submitters, each containing specific code to submit, monitor, cancel jobs on each of the supported DCIs (e.g., UNICORE [21], LSF [22], Moab [23]). Figure 2 presents a schematic overview of the interaction between the DCI Bridge and other components.

Designing a workflow in gUSE entails two steps: creation of an abstract graph, and a node-per-node configuration of the concrete. Creation of the abstract graph is achieved via a Java WebStart [24] application that is launched from WS-PGRADE, but is executed on the user's computer. At this point, users are able to provide only application domain information. After saving the abstract graph, users would direct their web browser back to WS-PGRADE, open the concrete for editing, and configure each task comprising the workflow separately. The configuration entails details such as provision of the required command line arguments to execute each task. Since gUSE offers interfaces to several middlewares, it is possible to execute tasks of the same workflow on different DCIs. The possible complexity of workflows that could be executed by gUSE is reflected in the several available fields on the task configuration windows presented to the user while configuring the concrete (see Fig. 6). The two-step creation of workflows (i.e., creation of the abstract graph and creation/configuration of the concrete, as depicted in Fig. 6), combined with the steep learning 


\section{Graphical User Interface}

\section{WS-PGRADE}

\section{gUSE Services}

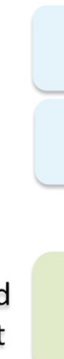

Workflow

Interpreter

Workflow Storage

File Storage

Application Repository

Information Service

\section{Data Avenue}

Fig. 1 The three-thiered gUSE's architecture. The three tiers of gUSE's architecture: WS-PGRADE acts as the user interface, the service layer handles e.g., file, workflow storage. The Job Submission and Data Management layer contains the DCI Bridge, which is responsible to access DCls. Figure based on [47]

curve that gUSE poses to new users is an aspect that might intimidate users without much technical experience.

Due to the diversity of workflow engines, a variety of workflow representations has arisen. This disparity of representations poses a challenge to scientists who desire to reuse workflows. Ideally, a scientist would design and test a workflow only once and it would be possible to execute it on any workflow engine on a given DCI. Built on this principle, the Sharing Interoperable Workflow for LargeScale Scientific Simulation on Available DCIs project [25]
(SHIWA) allows users to run previously existing workflows from different platforms on the SHIWA Simulation Platform. However, privacy concerns might give scientists second thoughts about executing their workflows and uploading their sensitive data on the SHIWA Simulation Platform. Tavaxy [8], focusing on genome comparison and sequence analysis, was created to enable the design of workflows composed of Taverna and Galaxy sub-workflows, and other workflow nodes in a single environment.

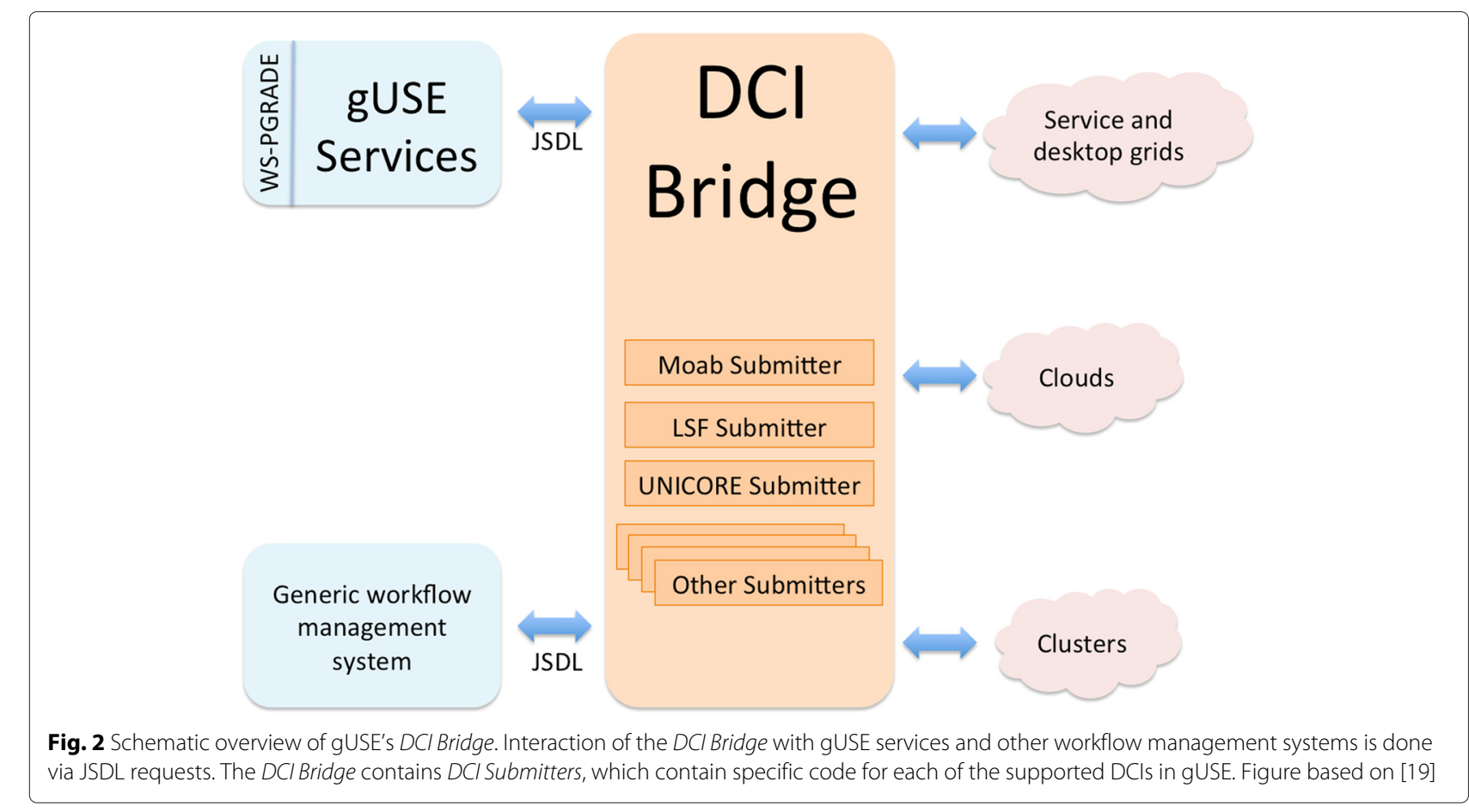


There is a clear overlap between the functionalities of the mentioned workflow engines. All offer royalty-free workflow design and execution. However, based on feedback from users and experience in our research group, we believe that the KNIME Analytics Platform is an accessible workflow editor, although it lacks on computing power. On the other hand, again based on experience and feedback, we see gUSE as a great back end framework that taps into several DCIs, but we have found that its workflow editing capabilities pose a steep learning curve to its user base.

In this paper we present work that bridges the gap between the KNIME Analytics Platform and gUSE. Our work will surely help adopters to significantly reduce the time spent designing, creating and executing repeatable workflows on distributed HPC resources.

\section{Workflow representation Formal representation}

Workflows can be represented using Petri nets [26, 27]. Petri nets are directed bipartite graphs containing two kinds of nodes: places and transitions.

A place represents a set of conditions that must be fulfilled for a transition to occur. Once a place has satisfied all its conditions, it is enabled. Transitions represent actions that affect the state of the system (i.e., copy a file, perform a computation, modify a file).

Places and transitions are connected by edges called arcs. No arc connects two nodes of the same kind (i.e., this restriction is precisely what makes Petri nets bipartite graphs). Arcs represent transitions' pre- and postconditions. In order for a transition to take place, all of its preceding places must be enabled (i.e., all of the conditions of preceding places must be satisfied). Conversely, when a transition has been completed, a postcondition is satisfied, influencing the state of subsequent places to which this transition is connected to.

Whenever a place's condition is satisfied, a token is depicted inside the corresponding place. Figure 3 depicts a simple computer process in which a molecule contained in an input file will be modified by adding any missing hydrogen atoms (i.e., the molecule will be protonated).

\section{High-level representation}

There are several alternatives to represent workflows in a platform-independent way. Yet another Workflow Language (YAWL) [28] was created after extensive analysis and review of already existing workflow languages in order to identify common workflow patterns and develop a new language that could combine the strengths and overcome the handicaps of other languages. The Interoperable Workflow Intermediate Representation (IWIR) [29] is a standard adopted by several workflow engines and is the language describing workflows in the SHIWA
Simulation Platform [25]. More recently a group of individuals, vendors and organizations joined efforts to create the Common Workflow Language (CWL) [30] in order to provide a specification that enables scientists to represent workflows for data-intensive scientific tasks (e.g., mass spectrometry analysis, sequence analysis).

For the sake of clarity and brevity, literature commonly depicts workflows as directed acyclical graphs, in which each vertex represents a place together with its pre- and postconditions (i.e., the preceding and following transitions) [31]. Each of the vertices is labelled, has a unique identifier and represents a task to be performed. Furthermore, each of the tasks in a workflow can receive inputs and can produce outputs. Outputs of a task can be channeled through another task as an input. An edge between two nodes represents the channeling of an output from a task into another. Edges determine the logical sequence to be followed (i.e., the origin task of an edge has to be completed before the destination task of an edge can be performed). A task will be executed once all of its inputs can be resolved. Workflow tasks are commonly referred by workflow systems as nodes or jobs and in this manuscript we will use these terms interchangeably.

\section{Workflow abstraction}

Workflows contain three different dimensions or abstraction layers, namely, case, process and resource dimensions [26]. Mapping these dimensions into concepts used on distributed execution workflow systems, we find that:

- The case dimension refers to the execution of a workflow (i.e., a single run).

- The process dimension, also referred to as the abstract layer [32, 33], deals with the application domain (i.e., the purpose of the workflow), therefore, technical details such as architecture, platform, libraries, implementation, programming language, etc., are hidden in this dimension.

- The resource dimension, often called the concrete layer [32, 33], encompasses the hardware and software used to execute the desired process; questions such as How many processors does a task require?, Which software running on which device will perform a certain task?, What are the provided parameters for a specific task?, etc., must be answered in this layer.

Given that the focus of our work is distributed workflows, we prefer the use of the abstract/concrete terminology throughout this document. Figure 4 depicts the abstract layer of the previously introduced protonation process. This workflow is now composed of four tasks (i.e., vertices) and three edges. The task labelled Input has no predecessor tasks, therefore this task is the first one to be executed. In comparison, the task labelled Protonate 


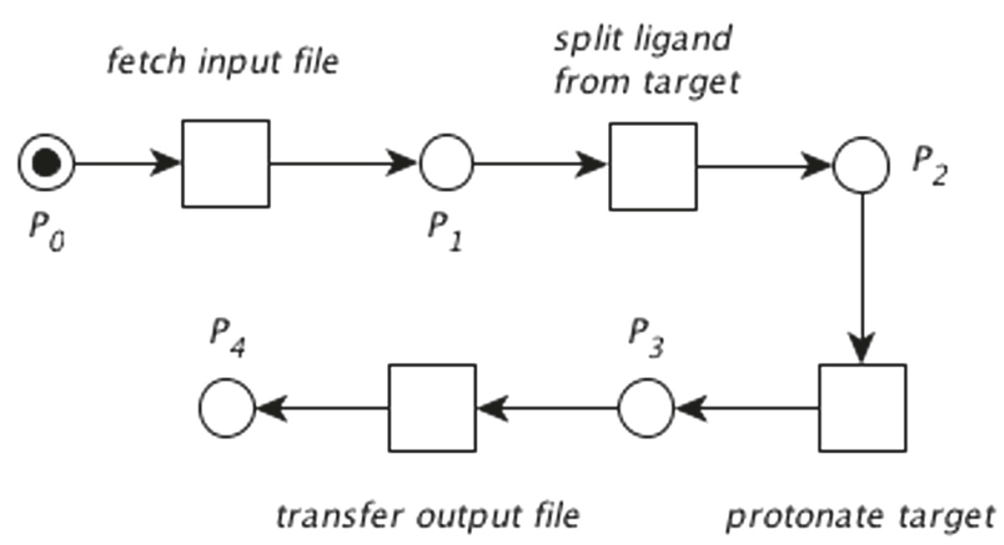

Fig. 3 A workflow as represented by a Petri net. Petri net modelling a software pipeline to protonate a molecule found in a single input file. Places are shown as circles, transitions are depicted as squares. The place $P_{0}$ expects and contains one token, represented by a black dot, and is thus enabled. It follows that $P_{0}$ is the starting place and $P_{4}$ represents the end of the process

depends on the completion of Split, which in turn depends on the completion of Input.

Figure 5, in contrast to Fig. 4, shows a possible concrete representation of the presented sample workflow, in which each vertex has been annotated with information needed in order to actually execute the corresponding tasks. While this information varies across workflow execution systems and platforms, the abstract representation of a workflow is constrained only to the application domain and is thus independent of the underlying software and infrastructure. At the concrete layer, the edges not only determine the logical sequence to be followed, but also represent channeled output files. For instance, the Protonate task receives an input file from its predecessor, Split and generates an output file that will be channeled to the Output task.

\section{Workflow conversion}

As it has been mentioned before, gUSE splits the creation of workflows into two steps, creation of the abstract graph and configuration of the concrete. These steps match the abstract and concrete layers we have discussed in the previous section. Both Galaxy and the KNIME Analytics Platform, in turn, represent workflows without this separation. In order to create a workflow in either Galaxy or the KNIME Analytics Platform, users drag and drop visual elements representing nodes into a workspace. Nodes come pre-configured and commonly execute a single task, therefore the user creates both the abstract and concrete layer at the same time. Inputs and outputs of nodes are visually represented and they can be connected to other nodes. Each node has a configuration dialog in which users can change the default parameters.

It is easy to see how functionally equivalent workflow constructs (e.g., conditional execution of a workflow section, parameter sweep, etc.) are represented differently across workflow engines. Furthermore, engines may offer features that are not available on other workflow systems (e.g., the KNIME Analytics Platform offers elements that are not present neither in gUSE nor in Galaxy, such as flow variables [34]). The proper identification and conversion of these elements is important for the conversion, since they play their part in workflow execution. Figure 6 displays a schematic comparison of the implementation of our example workflow across three selected workflow engines, the KNIME Analytics Platform, Galaxy, and gUSE.

Due to the variety of workflow systems-we have mentioned only a few instances, it is not surprising that there are several languages and file formats to represent workflows. A first step towards a successful conversion of workflows is to be able to represent the vertices of a workflow (i.e., the tasks, or nodes) in a consistent way across

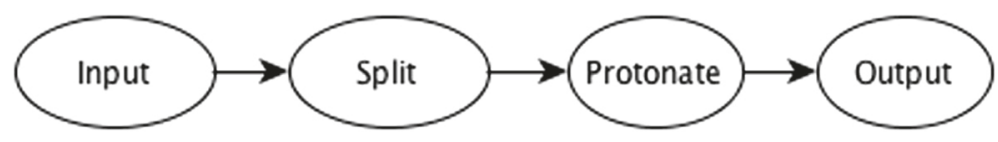

Fig. 4 An abstract workflow. Each vertex corresponds to a task and each edge corresponds to the logical sequence to be followed. Only application domain information is present 


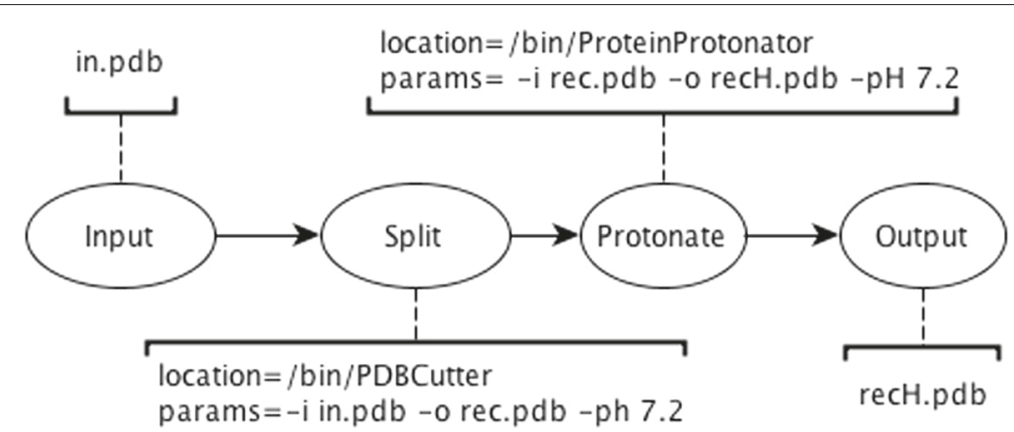

Fig. 5 A concrete workflow. Similar to an abstract workflow, a concrete workflow contains implicit application domain information. However, vertices of concrete workflows are annotated with extra attributes needed to actually execute the given tasks and obtain the needed data

platforms. Once a platform-independent representation of the vertices has been achieved, it is easier to import tasks into several workflow engines with less effort. Conversion of edges is an endeavour that is specific to each of the workflow engines (i.e., a task can be seen as a standalone component, while edges are the result of the collaboration of two or more tasks). Over the course of the last years, we have developed a series of tools enabling workflow interoperability across disparate workflow engines.

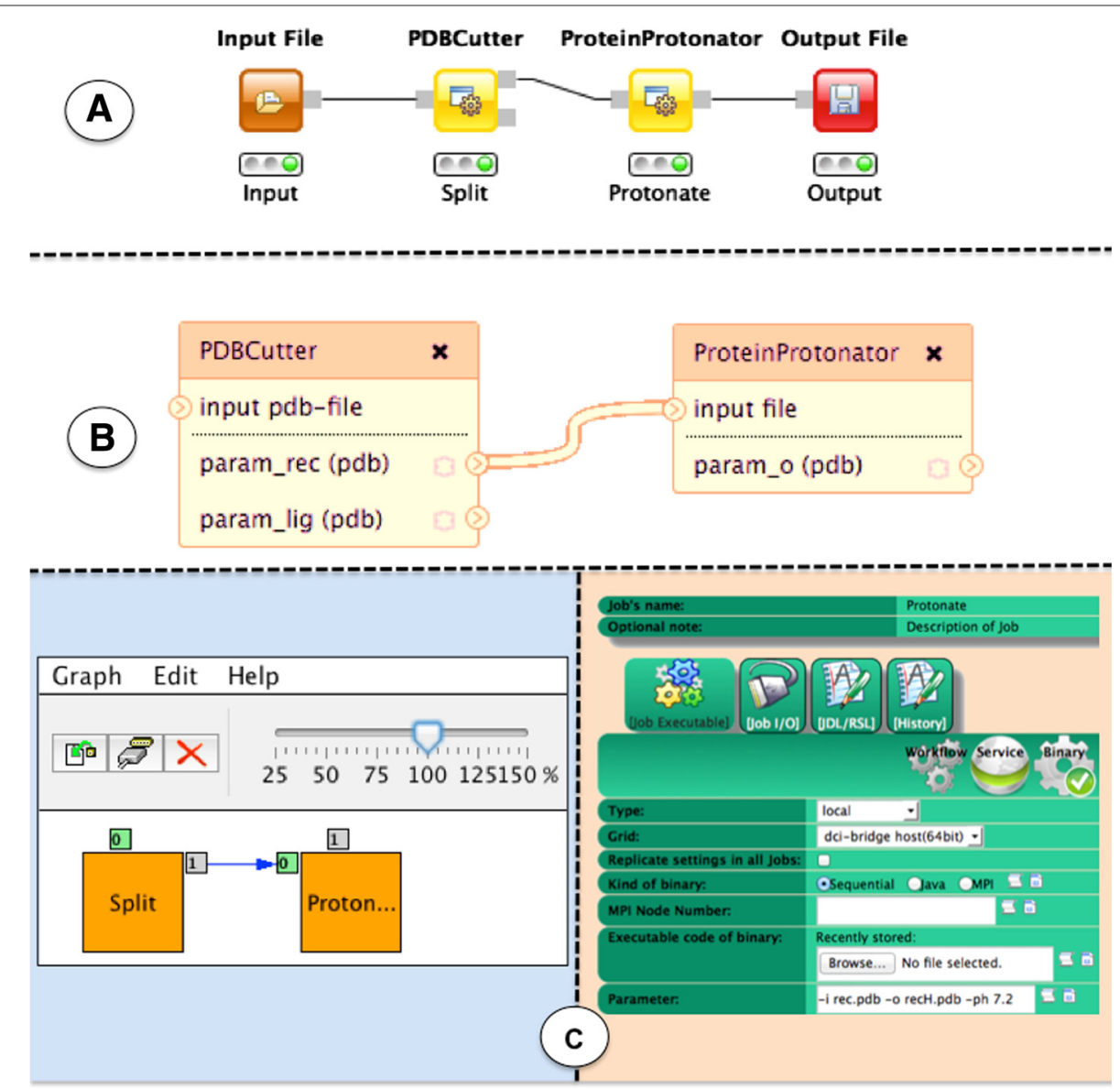

Fig. 6 Comparison of the same pipeline across KNIME Analytics Platform, Galaxy, gUSE. The KNIME Analytics Platform and Galaxy (sections A, B, respectively) offer an intuitive workflow creation and there is no perceived boundary between the abstract and the concrete layers. gUSE, however, (section C) splits the creation of workflows in two phases, creation of the abstract graph and the further configuration of each node in the concrete workflow 


\section{Implementation}

Conversion of whole workflows can be split into two parts, namely, conversion of vertices and conversion of edges. Vertices represent tasks that take inputs, parameters and produce outputs. This information can be represented in a structured way.

Common Tool Descriptor files (CTD) are XML documents that contain information about parameters, inputs and outputs of a given tool. This information is presented in a structured and human readable way, thus facilitating manual generation for arbitrary tools. Since CTDs are also properly formatted XML documents, it is a trivial matter to parse them in an automated way. Generation of CTDs can be either done manually or by CTD-enabled programs. Software libraries and suites such as SeqAn [35], OpenMS [36] and BALL [37] are CTD-enabled, that is, they are able to generate CTDs for each of its tools, parse input CTDs and execute tools accordingly. Executing a CTD-enabled tool across different platforms is a process transparent for end users. Figures 7 and 8 display how CTDs encapsulate needed runtime information into a single file, and how CTDs interact with other languages and platforms, respectively.

We have also worked towards making CTDs more accessible. Taking into account that refactoring tools to make them CTD-enabled might be time consuming, we have developed CTDopts in order to bind an already existing tool via Python wrappers. Naturally, this interaction is not done automatically without any further input, but this is an easier endeavor than performing a refactoring. CTDopts acts as a wrapper allowing users to execute arbitrary command line tools via CTDs. These Python wrappers will communicate directly with the tool, thus offering end users an interface to a CTD-enabled tool. Of course, manual creation of CTDs is always an option. Since CTDs are XML documents, a text editor is all is needed to manually generate them.

Representing a simple vertex using CTDs brings users closer to workflow interoperability, but this exercise might not pay off on its own. In order to extend a workflow engine by adding new tools, one could delve into the inner-workings of said workflow engine and import new tools. Since this could be a technical effort not accessible to users without the needed experience, we have also developed converters of tasks (i.e., vertices).

The KNIME Analytics Platform is an application based on the Eclipse Modelling Framework (EMF) [38], allowing the development of extensions. One of these extensions, and perhaps the most interesting for KNIME Analytics Platform users, is the ability to develop new KNIME nodes [14]. It is also possible to download so-called community nodes [15].

We developed the Generic KNIME Nodes (GKN) extension to make use of KNIME Analytics Platform's extensibility. GKN takes a set of CTDs as an input and generates the needed resources to implement KNIME nodes. In order to achieve this, its functionality is split into two main components: node generation and node execution. Once a node built via GKN has been generated (i.e., via the node generation component) and imported into the KNIME Analytics Platform, it interacts with other KNIME nodes via the node execution component. This interaction is transparent for the user.

Since Galaxy is one of the most popular workflow systems in the bioinformatics community, we felt that providing a suitable conversion would benefit the scientific community, so we developed CTD2Galaxy, a set of scripts to convert a CTD into a Galaxy ToolConfig XML file [9]. We also analysed Galaxy's toolshed [10] and we determined that it would be possible to automatically convert around 1200 of these tool descriptions into CTD files. The rest of the tools in the toolshed contain elements that are not easily translated and are not supported in CTD format (e.g., if-else constructs, for loops, etc.).

So far, we have discussed conversion of vertices. Different workflow engines represent workflows using a different format. It follows that conversion of edges is an effort that heavily depends on the involved workflow engines. Analog to the need of a platform-independent representation of vertices, a first step of workflow interoperability,

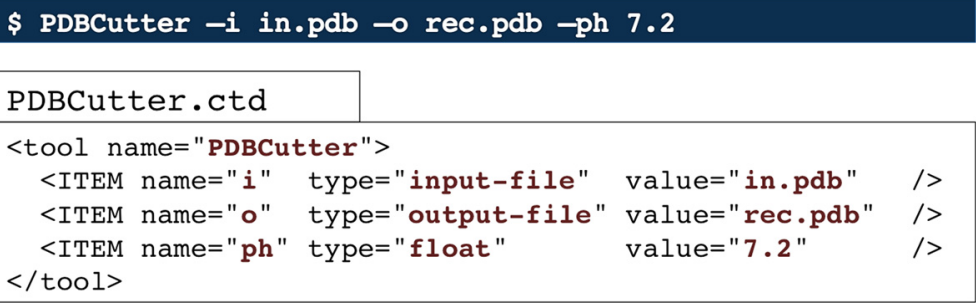

\$ PDBCutter -par PDBCutter.ctd

Fig. 7 A CTD in action. The upper section shows all three parameters needed for the tool PDBCutter to be executed. The middle section shows a snippet of a CTD representing a CTD-enabled tool. The bottom section shows how to execute a CTD-enabled tool with the given sample CTD 


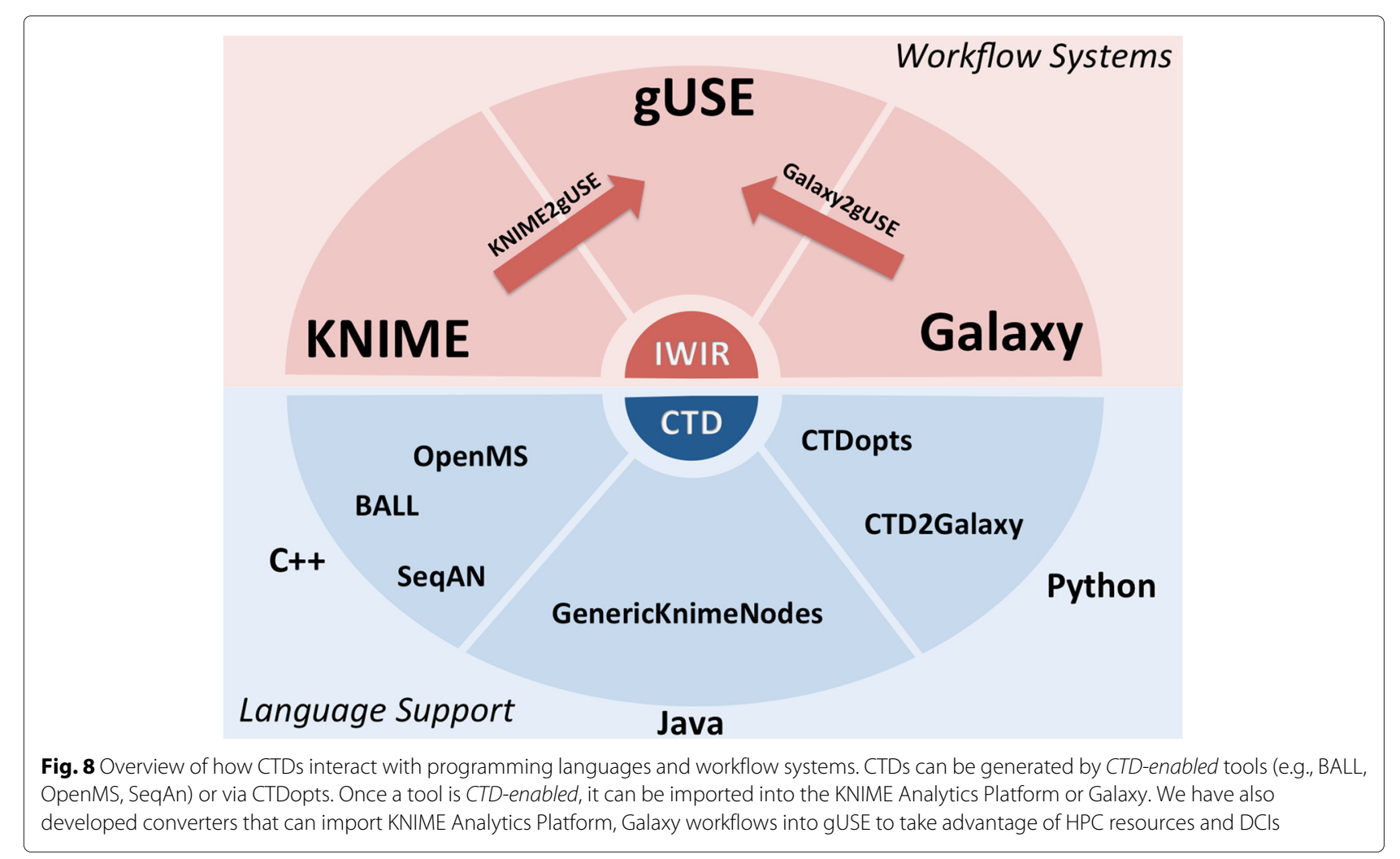

which is our end target, is the development of a platformindependent representation of workflows.

In spite of the apparent variety of workflow languages, we have focused our efforts in using the KNIME Analytics Platform as the source point of workflow conversion. To this end, we have also started work that could translate any KNIME Analytics Platform workflow into either an IWIR representation or a gUSE workflow (KNIME2gUSE). Alternatively, we have also developed a set of scripts that convert Galaxy workflows into gUSE (Galaxy2gUSE). Refer to Fig. 8 for a brief overview of how our work fits together against workflow systems.

\section{Results and discussion}

We have implemented a Label-free-quantification (LFQ) $[39,40]$ workflow in the KNIME Analytics Platform. LFQ is a widely used type of experiment in mass spectrometry based proteomics aimed at quantifying and comparing the abundances of peptides and proteins across different samples. Unlike other quantification strategies employing various kinds of chemical labelling of the different samples, LFQ does not impose a limit on the number of samples. Experiments with tens or hundreds of samples are routinely performed in many labs and, considering the ever-increasing performance of modern mass spectrometers, the number of samples to be analyzed per experiment is very likely to keep growing. This, in turn, gives rise to major computational challenges when analyzing the resulting large and complex data sets consisting of up to several terabytes of raw data. Hence, data processing and analysis of label-free quantification experiments can greatly benefit from distributed HPC resources and shall therefore serve as an example use case.

Our example workflow is based on tools provided by OpenMS/TOPP [36, 41-43]. In addition to label-free quantification, it performs a complete consensus peptide identification [44] using the search engines OMSSA [45] and X!Tandem [46]. In essence, so-called tandem mass spectra containing the masses of fragment ions resulting from collision-induced dissociation of selected peptides are compared to theoretical fragment spectra generated from a given FASTA database containing protein sequences. Afterwards, peptide hits are filtered so that the remaining set of identifications has a false discovery rate of less than $1 \%$. The quantification part starts with two major steps of data reduction and signal detection: peak picking and feature finding. Subsequently, the results of the identification and quantification branches of the workflow are combined, and corresponding peptide signals are matched across all samples in a process called feature linking. Finally, a normalization step is performed, which is necessary in order to be able to actually compare the relative abundances of peptides across the different runs. It is important to note that each run is executed independently 
via parameter sweep. Furthermore, each run is represented in a different input file, as given by the Input Files element. The output of the complete workflow is channeled to the TextExporter tool, which in turn generates a single comma-separated values (CSV) file containing all identified peptides together with their abundances in all given samples.

Figure 9 depicts the implementation of our LFQ workflow. We used our KNIME2gUSE extension and successfully imported our workflow in gUSE, as Fig. 10 shows.

\section{Conclusions}

Throughout this document we have presented our work towards workflow interoperability. We are convinced that investing time and effort in workflow interoperability helps scientists from all fields to expedite retrieval of results, so we tested and analyzed several workflow engines.

Based on user feedback and our own usage experience, we noticed that the creation of workflows in the KNIME Analytics Platform is straightforward, rapid and userfriendly. The needed amount of previous knowledge of the
KNIME Analytics Platform or other workflow systems to put together a workflow and execute it is minimal. However, we were not satisfied with the fact that execution of workflows on distributed HPC resources is royalty-based. Our search then brought us to gUSE.

gUSE is an open-source web-based framework that enables users to execute workflows on distributed HPC resources. It supports several major resource managers and middlewares via the use of so-called DCI Submitters, which can also be added to extend gUSE's support. However, workflow creation in gUSE is not as straightforward as in the KNIME Analytics Platform.

It was apparent that there was a need for a solution that combined the features and overcame the drawbacks of these two framework systems. On one side, a free and easy-to-use workflow editor and on the other side, a free and powerful back-end system connecting to several distributed HPC resources.

We are confident that our work presented in this document, in particular KNIME2gUSE, not only provides scientists a way to design and test workflows on their desktop computers, but also enables them to use powerful

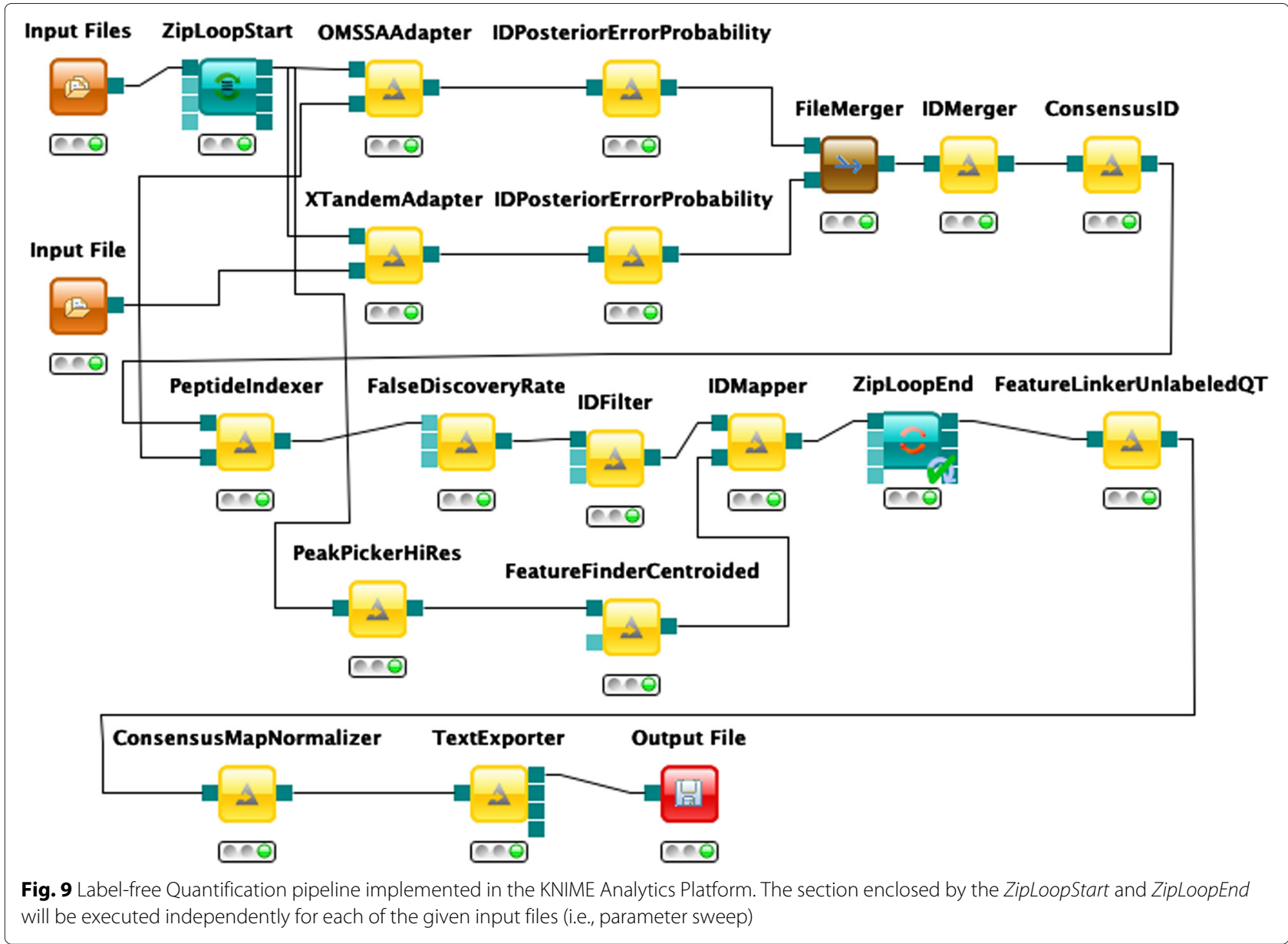




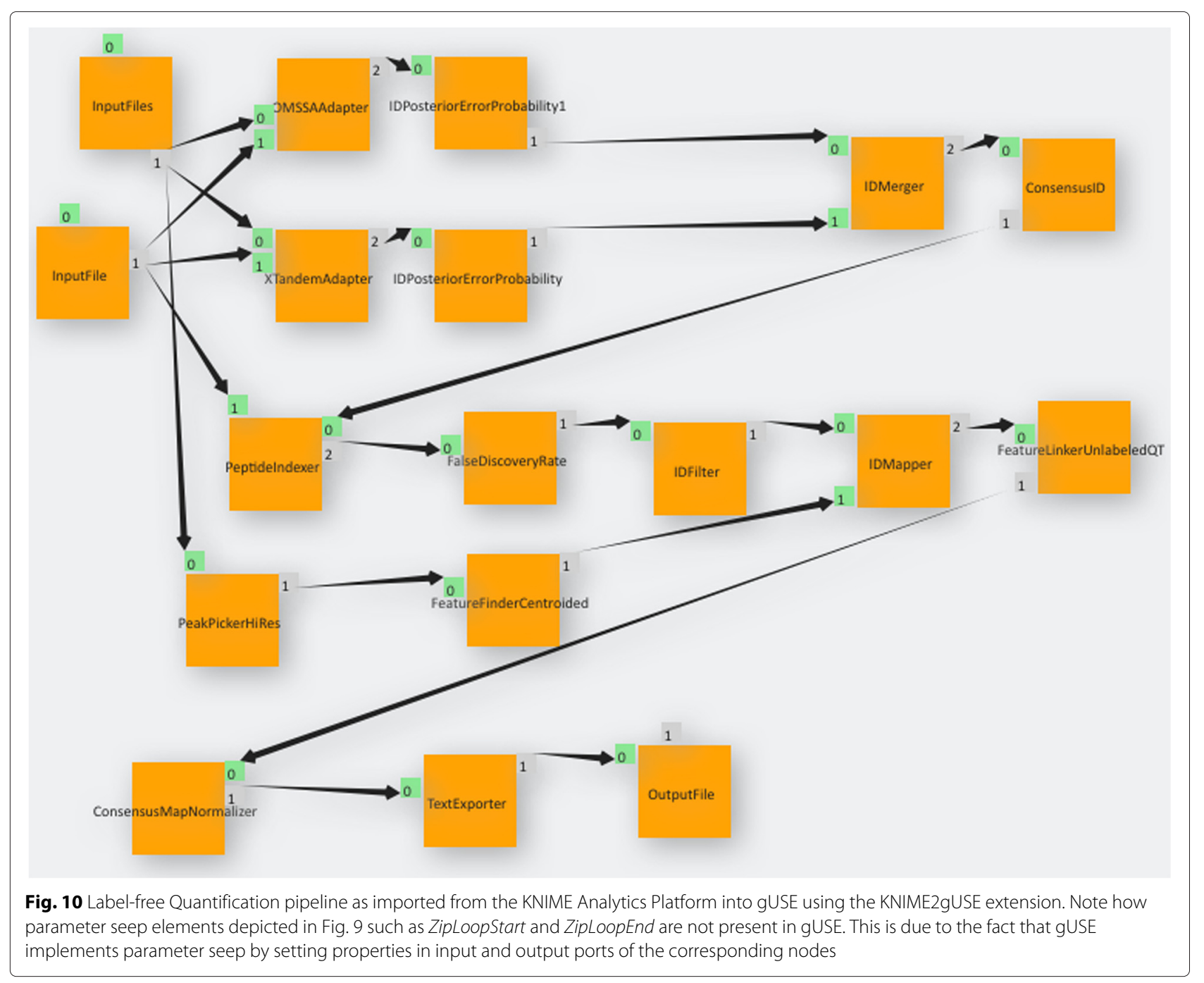

resources to execute their workflows, thus producing scientific results in a timely manner. We see KNIME2gUSE as a potential adopter of CWL: KNIME2gUSE could be extended in order to generate a CWL representation of a KNIME Analytics Platform workflow.

\section{Availability and requirements}

- Project name: Workflow Conversion.

- Project home page: http://workflowconversion. github.io/

- Operating system(s): Platform independent.

- Programming language: Java, Python.

- Other requirements: e.g. Python 2.7, Java 1.6 or higher.

- License: e.g. GNU General Public License (GPL).

- Any restrictions to use by non-academics: none.

\section{Abbreviations}

API: Application programming interface; BALL: Bioinformatics algorithms library: CSV: Comma separated values: CTD: Common tool descriptor; CWL: Common workflow language; DCl: Distributed computing infrastructure;
EMF: Eclipse modelling framework; GKN: Generic KNIME nodes; gUSE: Grid and cloud user support environment; HPC: High-performance computing; IWIR: Interoperable workflow intermediate representation; JSDL: Job submission description language; KNIME: Konstanz information miner; LFQ: Label-ree quantification; LSF: Platform load sharing facility; OPM: Open provenance model; OMSSA: Open mass spectrometry search algorithm; SHIWA: Sharing interoperable workflow for large-scale scientific simulation on available DCls project; TOPP: The OpenMS proteomics pipeline; UNICORE: Uniform interface to computing resources; WSDL: Web services description language; WSPGRADE: Web services parallel grid runtime and developer environment portal; XML: Extensible markup language; YAWL: Yet another Workflow Language.

\section{Competing interests}

We declare that we have no competing interests.

\section{Authors' contributions}

LdlG wrote the manuscript, carried out development work for KNIME2gUSE, CTD2Galaxy and contributed to GKN, CTDopts. JV created, tested the LFQ pipeline in the KNIME Analytics Platform and was involved in drafting the manuscript. SA, together with MR developed the GKN extension. AS has been a major contributor and released the first version of CTDopts. LdIG, SA, MR, AS, $\mathrm{OK}$ and $\mathrm{KR}$ are responsible for the development and maintenance of CTD schemas. SG released the first version of Galaxy2gUSE. OK, KR conceived the study, participated in its design and coordination, and helped to draft the manuscript. All authors read and approved the final manuscript. 


\section{Authors' information}

We have no additional information about the authors.

\section{Acknowledgements}

The authors would like to thank Bernd Wiswedel, Thorsten Meinl, Patrick Winter and Michael Berthold for their support, patience and help in developing the KNIME2gUSE extension. Supported by the German Network for Bioinformatics Infrastructure (Deutsches Netzwerk für Bioinformatik-Infrastruktur, de.NBI). We acknowledge support by Deutsche Forschungsgemeinschaft and Open Access Publishing Fund of University of Tübingen.

\section{Author details}

${ }^{1}$ Center for Bioinformatics and Dept. of Computer Science, University of Tübingen, Sand 14, 72070 Tübingen, Germany. ${ }^{2}$ Algorithmic Bioinformatics, Computer Science Institute, Freie Universität Berlin, Takustr. 9, 14195 Berlin, Germany. ${ }^{3}$ College of Engineering, University of Notre Dame, 257 Fitzpatrick Hall, 46556 Notre Dame, IN, United States.

\section{Received: 12 November 2015 Accepted: 3 March 2016 Published online: 12 March 2016}

\section{References}

1. Gratzer W. Trouble at the lab. Economist. 2013;302(5911):774-5. doi:10.1038/302774a0.

2. McNutt M. Reproducibility. Science (New York, N.Y.) 2014;343(6168):229 doi:10.1126/science.1250475.

3. Greene CS, Tan J, Ung M, Moore JH, Cheng C. Big data bioinformatics. J Cell Physiol. 2014;229(12):1896-900. doi:10.1002/jcp.24662.

4. Berthold MR, Cebron N, Dill F, Gabriel TR, Kotter T, Meinl T, Ohl P, Sieb C, Thiel K, Wiswedel B. Knime. Web. 20071-8. doi:10.1007/978-3540-78246-9.

5. Kacsuk P, Farkas Z, Kozlovszky M, Hermann G, Balasko A, Karoczkai K, Marton I. WS-PGRADE/gUSE generic DCI gateway framework for a large variety of user communities. J Grid Comput. 2012;10(4):601-30. doi:10.1007/s10723-012-9240-5.

6. Blankenberg D, Kuster GV, Coraor N, Ananda G, Lazarus R, Mangan M Nekrutenko A, Taylor J. Galaxy: A web-based genome analysis tool for experimentalists. 2010. NIHMS150003. doi:10.1002/0471142727. mb1910s89.

7. Missier P, Soiland-Reyes S, Owen S, Tan W, Nenadic A, Dunlop I, Williams A, Oinn T, Goble C. Taverna, reloaded. In: Lecture Notes in Computer Science (including Subseries Lecture Notes in Artificial Intelligence and Lecture Notes in Bioinformatics), vol. 6187 LNCS; 2010. p. 471-81. doi:10.1007/978-3-642-13818-8_33.

8. Abouelhoda M, Issa S, Ghanem M. Tavaxy: Integrating Taverna and Galaxy workflows with cloud computing support. 2012. doi:10.1186/1471-2105-13-77.

9. Galaxy Tool XML File. https://wiki.galaxyproject.org/Admin/Tools/ ToolConfigSyntax. Accessed 28 July 2015.

10. Galaxy Tool Shed. https://toolshed.g2.bx.psu.edu/. Accessed 07 July 2015.

11. Moreau L, Clifford B, Freire J, Futrelle J, Gil Y, Groth P, Kwasnikowska N, Miles S, Missier P, Myers J, Plale B, Simmhan Y, Stephan E, Den Bussche $J V$. The Open Provenance Model core specification (v1.1). In: Future Generation Computer Systems, vol. 27; 2011. p. 743-56. doi:10.1016/j.future.2010.07.005

12. Goble CA, Bhagat J, Aleksejevs S, Cruickshank D, Michaelides D, Newman D, Borkum M, Bechhofer S, Roos M, Li P, de Roure D. myExperiment: A repository and social network for the sharing of bioinformatics workflows. Nucleic Acids Res. 2010;38(SUPPL. 2). doi:10.1093/nar/gkq429.

13. KNIME | Open for Innovation. http://www.knime.org/. Accessed 29 June 2015.

14. KNIME | New Node Wizard. https://tech.knime.org/new-node-wizard. Accessed 06 July 2015.

15. KNIME | Community Contributions. https://tech.knime.org/community. Accessed 07 July 2015.

16. KNIME | KNIME Cluster Execution. https://www.knime.org/clusterexecution. Accessed 06 July 2015

17. KNIME | KNIME Server - The Heart of a Collaborative KNIME Setup. https:// www.knime.org/knime-server. Accessed 06 July 2015.
18. Web Service Definition Language (WSDL). http://www.w3.org/TR/wsdl. Accessed 06 July 2015.

19. DCl Administration Manual, Version 3.7.1. http://sourceforge.net/ projects/guse/files/3.7.1/Documentation/DCI_BRIDGE_MANUAL_v3.7.1. pdf/download.

20. Anjomshoaa A, Brisard F, Drescher M, Fellows D, Ly A, McGough S, Pulsipher D, Savva A. Job Submission Description Language (JSDL) Specification, Version 1.0. 20051-72. Open Grid Forum.

21. Romberg M. The UNICORE Grid Infrastructure. Spec Issue Grid Comput Scientifc Program J. 2002;10:149-57.

22. IBM Platform Computing Products: Workload Management Platform Platform LSF. IBM Corporation. 2012. http://www-03.ibm.com/systems/ platformcomputing/products/lsf/.

23. HPC Products - Adaptive Computing. http://www.adaptivecomputing. com/products/hpc-products/. Accessed 06 July 2015.

24. Java SE Desktop Technologies - Java Web Start Technology. http://www. oracle.com/technetwork/java/javase/javawebstart/index.html. Accessed 03 July 2015

25. Terstyanszky G, Kukla T, Kiss T, Kacsuk P, Balasko A, Farkas Z. Enabling scientific workflow sharing through coarse-grained interoperability. Futur Gener Comput Syst. 2014;37:46-59. doi:10.1016/j.future.2014.02.016.

26. van der Aalst WMP. The application of petri nets to workflow management. 1998. doi:10.1142/S0218126698000043.

27. Peterson JL, Vol. 24. Petri Net Theory and the Modeling of Systems; 1981, p. 290. doi:10.1016/0378-4754(82)90087-8.

28. van der Aalst WMP, ter Hofstede AHM. YAWL: yet another workflow language. Inf Syst. 2005;30(4):245-75. doi:10.1016/j.is.2004.02.002.

29. Plankensteiner K, Montagnat J, Prodan R. IWIR: A Language Enabling Portability Across Grid Workflow Systems. In: SIGMOD Rec; 2011. p. 97-106. doi:10.1145/2110497.2110509. http://doi.acm.org/10.1145/ 2110497.2110509 .

30. Common Workflow Language. http://www.oracle.com/technetwork/ java/javase/javawebstart/index.html. Accessed 03 July 2015.

31. Salimifard K, Wright M. Petri net-based modelling of workflow systems: An overview. 2001. doi:10.1016/S0377-2217(00)00292-7.

32. Deelman E, Blythe J, Gil Y, Kesselman C, Mehta G, Vahi K, Blackburn K, Lazzarini A, Arbree A, Cavanaugh R, Koranda S. Mapping abstract complex workflows onto grid environments. J Grid Comput. 2003;1(1): 25-39. doi:10.1023/A:1024000426962.

33. Yu J, Buyya R. A taxonomy of scientific workflow systems for grid computing. 2005. doi:10.1145/1084805.1084814

34. KNIME |Flow Variables. https://tech.knime.org/wiki/flow-variables. Accessed 26 Oct 2015.

35. Döring A, Weese D, Rausch T, Reinert K. SeqAn an efficient, generic C++ library for sequence analysis. BMC Bioinforma. 2008;9(1):11. doi:10.1186/ 1471-2105-9-11.

36. Sturm M, Bertsch A, Gröpl C, Hildebrandt A, Hussong R, Lange E, Pfeifer N, Schulz-Trieglaff O, Zerck A, Reinert K, Kohlbacher O. Openms an open-source software framework for mass spectrometry. BMC Bioinforma. 2008;9:163.

37. Hildebrandt A, Dehof AK, Rurainski A, Bertsch A, Schumann M, Toussaint NC, Moll A, Stöckel D, Nickels S, Mueller SC, Lenhof HP, Kohlbacher O. BALL-biochemical algorithms library 1.3. BMC Bioinforma. 2010;11:531. doi:10.1186/1471-2105-11-531.

38. Steinberg D, Budinsky F, Paternostro M, Merks E. EMF: Eclipse Modeling Framework; 2008, p. 744. doi:10.1108/02641610810878585. http://portal. acm.org/citation.cfm?id=1197540.

39. Bantscheff M, Schirle M, Sweetman G, Rick J, Kuster B. Quantitative mass spectrometry in proteomics: a critical review. Anal Bioanal Chem. 2007;389(4):1017-1031.

40. Weisser H, Nahnsen S, Grosman J, Nilse L, Quandt A, Brauer H, Sturm M, Kenar E, Kohlbacher O, Aebersold R, Malmstrom L. An automated pipeline for high-throughput label-free quantitative proteomics. J Proteome Res. 2013;12(4):1628-1644.

41. Kohlbacher O, Reinert K, Gröpl C, Lange E, Pfeifer N, Schulz-Trieglaff O, Sturm M. Topp-the openms proteomics pipeline. Bioinformatics. 2007;23(2):191-7.

42. Junker J, Bielow C, Bertsch A, Sturm M, Reinert K, Kohlbacher O. Toppas: a graphical workflow editor for the analysis of high-throughput proteomics data. J Proteome Res. 2012;11(7):3914-920. 
43. OpenMS | An Open-source Framework for Mass Spectrometry and TOPP - The OpenMS Proteomics Pipeline. http://open-ms.sourceforge.net/. Accessed 26 June 2015.

44. Nahnsen S, Bertsch A, Rahnenführer J, Nordheim A, Kohlbacher O. Probabilistic consensus scoring improves tandem mass spectrometry peptide identification. J Proteome Res. 2011;10(8):3332-343.

45. Geer LY, Markey SP, Kowalak JA, Wagner L, Xu M, Maynard DM, Yang X, Shi W, Bryant SH. Open mass spectrometry search algorithm. J Proteome Res. 2004;3(5):958-64.

46. Craig R, Beavis RC. Tandem: matching proteins with tandem mass spectra. Bioinformatics. 2004;20(9):1466-1467.

47. gUSE in a Nutshell. http://sourceforge.net/projects/guse/files/gUSE_in_ a_Nutshell.pdf/download.

\section{Submit your next manuscript to BioMed Central} and we will help you at every step:

- We accept pre-submission inquiries

- Our selector tool helps you to find the most relevant journal

- We provide round the clock customer support

- Convenient online submission

- Thorough peer review

- Inclusion in PubMed and all major indexing services

- Maximum visibility for your research

Submit your manuscript at www.biomedcentral.com/submit 